\title{
T Tauri Binaries in Orion: Evidence for Accelerated and Synchronized Disk Evolution
}

\author{
Sebastian Daemgen ${ }^{1}$, Monika G. Petr-Gotzens ${ }^{1}$, and Serge Correia ${ }^{2}$ \\ ${ }^{1}$ European Southern Observatory, Karl-Schwarzschildstr. 2, 85748 Garching, Germany \\ email: sdaemgen@eso.org \\ ${ }^{2}$ Astrophysikalisches Institut Potsdam, An der Sternwarte 16, 14482 Potsdam, Germany
}

\begin{abstract}
In order to trace the role of binarity for disk evolution and hence planet formation, we started the currently largest spatially resolved near-infrared photometric and spectroscopic study of the inner dust and accretion disks of the individual components of 27 visual, 100$400 \mathrm{AU}$ binaries in the Orion Nebula Cluster (ONC). We study the frequency of Brackett- $\gamma$ $(2.165 \mu \mathrm{m})$ emitters to assess the frequency of accretion disk-bearing stars among the binaries of the ONC: only $34 \pm 9 \%$ of the binary components show signs of accretion and, hence, the presence of gaseous inner disks - less than the fraction of gas accretion disks among single stars of the $\mathrm{ONC}$ of $\sim 50 \%$. Additionally, we find a significant difference between binaries above and below $200 \mathrm{AU}$ separation: no close systems with only one accreting component are found. The results suggest shortened disk lifetimes as well as synchronized disk evolution.
\end{abstract}

Keywords. stars: late-type, stars: formation, circumstellar matter, binaries: visual

\section{The Project \& Observations}

Twenty-seven binaries in the ONC were selected from the binary census in Petr(1998) and Köhler et al. (2006). The sample consists primarily of low-mass stars with spectral types K3-M6; separations are in a range of $0.25^{\prime \prime}-1^{\prime \prime}$, which is equivalent to $\sim 100$ $400 \mathrm{AU}$ at the distance of the ONC. All binaries were spatially resolved with Adaptive Optics assisted VLT/NACO+Gemini/NIRI Near-Infrared ( $J H K)$ photometry and $(K$ band, $2.2 \mu \mathrm{m}$ ) spectroscopy. Stellar parameters (spectral types, ages, masses, luminosities, and others) and dust disk and accretion signatures (Brackett- $\gamma$ equivalent widths, NIR color excess, accretion luminosities, mass accretion rates) were derived for each binary component. Active accretion from the inner disk around each stellar component was inferred from the presence of emission in the Brackett- $\gamma(2.166 \mu \mathrm{m})$ Hydrogen line, the presence of dust in the inner disk is measured from color excess in $H-K$.

\section{Results}

We find an accretion disk fraction of $34 \pm 9 \%$ among the ONC binary components. This is lower than the $50 \%$ accretion fraction found for singles in the ONC (Hillenbrand et al. 1998). We also see a reduced - compared to single stars in the ONC - number of binary components with $H-K$ color excess. A reason for this deficiency might be accelerated disk dispersal: since the disks of binary components are truncated to radii of $\sim 0.3-0.5$ times the binary separation (Armitage et al. 1999), viscous evolution at the outer radius of the disk can reduce the disk lifetime to scales $\ll 1 \mathrm{Myr}$.

At separations smaller than $200 \mathrm{AU}$, we see a relatively high abundance of pairs of two classical T Tauri stars (with both components accreting) and two weak-line T Tauri stars 


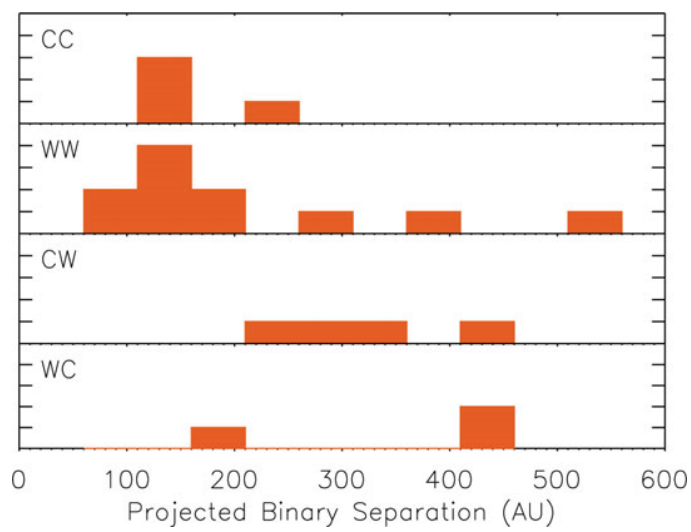

Figure 1. Histograms of binary separation as a function of component accretion activity: Pairs of classical T Tauri Stars (CC) and pairs of weak-line T Tauri Stars (WW) appear in tighter binary systems than mixed pairs with classical primary and weak-lined secondary $(\mathrm{CW})$ or the other way around (WC). This is statistically significant (K-S test) with $99.5 \%$.

(no accreting component; see Fig. 1). In contrast, mixed pairs of accreting/non-accreting components have significantly larger separations with $99.5 \%$ confidence (K-S test). Hints for an under-density of close mixed pairs have been observed in other star forming regions (Monin et al. 2007), however, never with such high significance. A possible explanation: independent disk evolution in wide pairs while disks in close binaries are synchronized.

The mass accretion rates of binary components of the ONC are comparable to those of other star forming regions, in the same mass range. Despite the considerably denser stellar environment of the ONC and reduced disk masses compared to star forming regions, like e.g. Taurus, the magnitude of accretion from the inner disk is not significantly reduced.

\section{Discussion}

Our results suggest shortened disk lifetimes and provide statistically significant evidence for synchronized evolution of the individual disks around medium-separated binary components - with important implications for planet formation scenarios. For instance, among the more than 40 planets in orbit around a stellar binary component (Eggenberger \& Udry 2010), it is mainly the more massive component hosting the planet. While this could be explained by shorter-lived disks around the less massive binary components, in our data, mixed systems with either component hosting a disk are equally abundant. Likewise, only few binaries with each component orbited by its own planet are known. If this is not entirely due to selection effects, it will most likely be a consequence of differential disk evolution, which we, however, observe to be attenuated in $<200 \mathrm{AU}$ binaries.

\section{References}

Petr 1998, PhDT, U Heidelberg, 1998

Köhler, R., Petr-Gotzens, M. G., McCaughrean, M. J., Bouvier, J., Duchêne, G., Quirrenbach, A., \& Zinnecker, H. 2006, A\& $A, 458,461$

Hillenbrand, L. A., Strom, S. E., Calvet, N., Merrill, K. M., Gatley, I., Makidon, R. B., Meyer, M. R., \& Skrutskie, M. F. 1998, AJ, 116, 1816

Armitage, P. J., Clarke, C. J., \& Tout, C. A. 1999, MNRAS, 304, 425

Monin, J.-L., Clarke, C. J., Prato, L., \& McCabe, C. 2007, PPV, 295

Eggenberger \& Udry 2010, in Planets in Binary Systems, ed: Haghighipour, Springer 2010 